\title{
Modeling and Control of a Dual Stage Actuator Hard Disk Drive*
}

\author{
Uwe BOETTCHER ${ }^{* *}$, Raymond A. DE CALLAFON** and Frank E. TALKE ${ }^{* *}$ \\ ** University of California, San Diego \\ Center for Magnetic Recording Research \\ 9500 Gilman Drive \\ La Jolla, CA 92093-0401, U.S.A \\ E-mail: uwe@ucsd.edu
}

\begin{abstract}
A data-based approach is presented for modeling and controller design of a dual-stage servo actuator in a hard disk drive. The servo actuator in this hard disk drive consists of a conventional voice coil motor and a piezo-electrically actuated suspension. A weighted Hankel matrix based realization algorithm that uses frequency domain data is applied to estimate a discrete-time model of the voice coil motor and the piezoelectric actuator. Based on the discrete-time models, different dual-stage track-following controllers were designed using classic and $H_{\infty}$ loop shaping techniques. The controllers were implemented in real-time in the investigated hard disk drive. A stable feed-back control and good agreement between measurements and simulations show the promising result of data based modeling and control.
\end{abstract}

Key words : Modeling, Positioning, Piezo-Element, Servo Mechanism, Motion Control

\section{Introduction}

Hard disk drives have improved enormously in terms of storage capacity, data access time and miniaturization over the last couple of decades, although their main functional principle has not changed substantially. Figure 1 shows a commercially available hard disk drive (HDD) that contains a dual-stage actuated suspension. As storage density increases, dualstage actuation is believed to be a solution in order to meet the higher accuracy and speed requirements on the servo mechanism in a $\mathrm{HDD}^{(1)}$ and during the process of servo writing $^{(2)}$. A number of different approaches for a second stage actuator in a hard disk drive

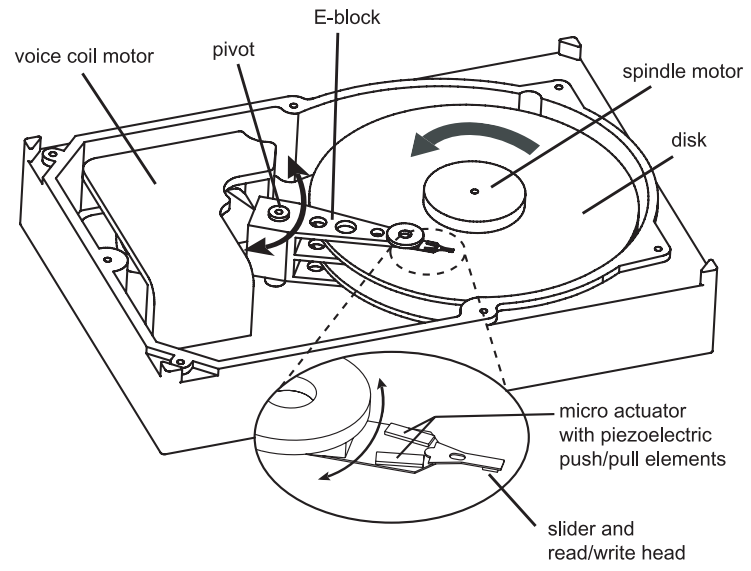

Fig. 1 Schematic of the investigated dual-stage actuated hard disk drive.

${ }^{*}$ Received 30 July, 2009 (No. 09-0395) [DOI: 10.1299/jamdsm.4.107]

Copyright (c) 2010 by JSME servo system have been explored within the last few years. Some of these micro-actuators are MEMS-type devices (microelectromechanical systems) based on electrostatic or electromagnetic effects ${ }^{(3)-(10)}$. Other micro-actuators are based on piezoelectric principles ${ }^{(11)-(13)}$. The 
actuators are either mounted on the suspension or on the slider. There are also approaches that have the micro-actuator integrated in the slider and allow a flying height adjustment in addition to off-track positioning ${ }^{(14)}$. A good overview of available second-stage actuators and control schemes is given in ${ }^{(15)}$. The second-stage actuator can also be used for active vibration damping using self sensoring ${ }^{(16),(17)}$. The dual-stage hard disk drive considered in this paper uses a push/pull actuator based on a piezoelectric transducer (PZT). The magnified portion of Fig. 1 shows a micro-actuator mounted on a suspension. A close-up of the slider with the suspension and the PZT elements is shown in Fig.2. The micro-actuator has a limited stroke, and, thus, it is more useful for track-following than for track-seeking, however, it can be used for short distance seeks ${ }^{(18),(19)}$.

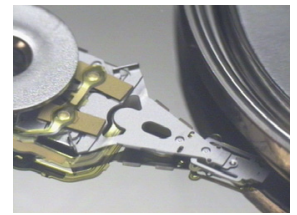

Fig. 2 Piezo-electric push/pull actuated suspension in a commercial available hard disk drive.

\section{Modeling and system identification}

In order to be able to inject control signals, the hard disk drive servo controller is bypassed completely. The circuit board was disconnected from the HDD and all motor drivers were replaced. Since the position error signal (PES) of the servo mechanism is not directly available, a laser Doppler vibrometer (LDV) is used to measure the radial slider motion. To accomplish visual access to the slider the HDD must be modified. The top cover was replaced with one made out of plexiglas and a mirror was used to deflect the laser beam onto the side of the slider. The experimental set-up illustrated in Fig. 3 was used to determine the frequency response function of both actuators.

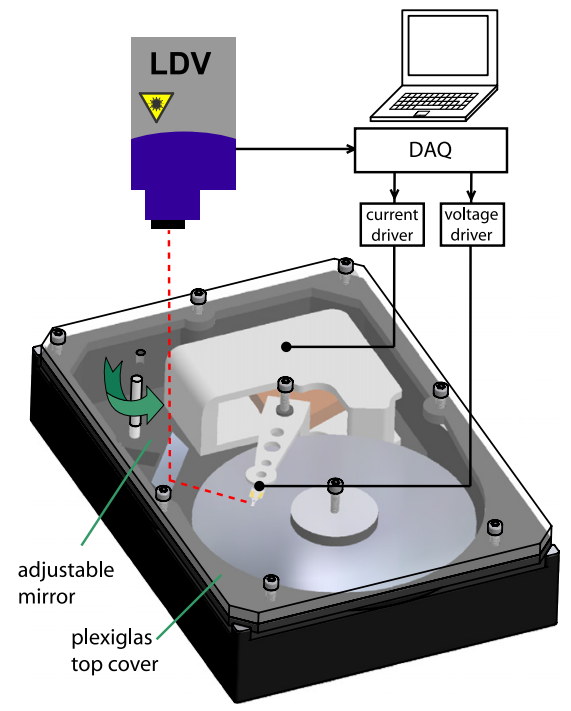

Fig. 3 Schematic of the experimental set-up to determine the frequency response function of the dual-stage actuator.

Many modeling approaches yield a continuous time model of the actuator ${ }^{(18),(20)}$, however, we estimate directly a discrete-time model using the eigensystem realization algorithm (21). It uses frequency domain data that is converted into time domain data first. Additional frequency-dependent weighting functions are used to emphasize control relevant resonance modes of the actuator response. The inverse discrete Fourier transform (IDFT) of the frequency response function (FRF) measurement yields an estimate for the impulse response of 
the system. The impulse response coefficients (Markov parameters) are defined by

$$
g_{k}=\frac{1}{2 N} \sum_{l=0}^{2 N-1} G_{l} e^{j \omega_{k} l}, \quad k=0,1, \cdots, 2 N-1
$$

where $G_{l}$ contains the FRF data and $\omega_{k}$ is the frequency vector defined by

$$
\omega_{k}=\frac{\pi k}{N}, \quad k=0,1, \cdots, 2 N-1
$$

$N$ denotes the number of FFT lines (frequency points) in the FRF measurements. The measured data are stored in a Hankel matrix $\mathbf{H}$ that contains the Markov parameter estimates defined in (1). By choosing $m$ as the number of impulse response samples taken into account, one can define a $m \times m$ Hankel matrix by

$$
\mathbf{H}=\left[\begin{array}{cccc}
g_{1} & g_{2} & \cdots & g_{m} \\
g_{2} & g_{3} & \cdots & g_{m+1} \\
\vdots & \vdots & \vdots & \vdots \\
g_{m} & g_{m+1} & \cdots & g_{2 m-1}
\end{array}\right]
$$

The shifted version $\overline{\mathbf{H}}$ is defined by

$$
\overline{\mathbf{H}}=\left[\begin{array}{cccc}
g_{2} & g_{3} & \cdots & g_{m+1} \\
g_{3} & g_{4} & \cdots & g_{m+2} \\
\vdots & \vdots & \vdots & \vdots \\
g_{m+1} & g_{m+2} & \cdots & g_{2 m}
\end{array}\right]
$$

To perform a control orientated modeling by means of capturing relevant resonance modes only, an input weighting filter $F_{u}$ is used. Performing an IDFT on $F_{u}$ yields

$$
g_{u_{k}}=\frac{1}{2 N} \sum_{l=0}^{2 N-1} F_{u_{k}} e^{j \omega_{k} l}, k=0,1, \cdots, 2 N-1
$$

The impulse response of the weighting filter $g_{u_{k}}$ is stored in a $N \times N$ Toeplitz matrix defined by

$$
\boldsymbol{\Gamma}_{\mathbf{u}}=\left[\begin{array}{cccc}
g_{u_{0}} & g_{u_{1}} & \cdots & g_{u_{N-1}} \\
0 & g_{u_{0}} & \cdots & g_{u_{N-2}} \\
\vdots & \vdots & \vdots & \vdots \\
0 & 0 & \cdots & g_{u_{0}}
\end{array}\right]
$$

The procedure used in this paper has been previously reported in $^{(22)}$ and ${ }^{(23)}$. The singular value decomposition (SVD) is applied to the weighted Hankel matrix $\mathbf{H}_{\mathbf{w}}$ defined by

$$
\mathbf{H}_{\mathbf{w}}=\mathbf{H} \boldsymbol{\Gamma}_{\mathbf{u}}=\mathbf{U} \boldsymbol{\Sigma} \mathbf{V}^{\mathrm{T}}
$$

where $\mathbf{V}, \mathbf{U}$ and $\boldsymbol{\Sigma}$ represent the unitary matrices and the singular value matrix of a standard SVD. The SVD is used to reduce $\mathbf{H}_{\mathbf{w}}$ to a matrix with rank $n$

$$
\mathbf{H}_{\mathrm{wn}}=\mathbf{H}_{1} \mathbf{H}_{2}
$$

where $\mathbf{H}_{1}$ and $\mathbf{H}_{2}$ are defined by

$$
\mathbf{H}_{1}=\mathbf{U}_{\mathrm{n}} \boldsymbol{\Sigma}_{\mathrm{n}}^{\mathbf{1 / 2}}, \quad \mathbf{H}_{2}=\boldsymbol{\Sigma}_{\mathrm{n}}^{1 / 2} \mathbf{V}_{\mathrm{n}}^{\mathrm{T}}
$$

An estimation for the state space matrix $\mathbf{A}$ is

$$
\mathbf{A}=\mathbf{H}_{1}^{*} \overline{\mathbf{H}}_{\mathbf{w}} \mathbf{H}_{2}^{*}
$$

where $\mathbf{H}_{1}^{*}$ and $\mathbf{H}_{2}^{*}$ denote the left and right inverse of $\mathbf{H}_{\mathbf{1}}$ and $\mathbf{H}_{2}$, respectively. The input matrix $\mathbf{B}$ becomes the first column of $\mathbf{H}_{2} \boldsymbol{\Gamma}_{\mathbf{u}}{ }^{-1}$. The first row of $\mathbf{H}_{\mathbf{1}}$ forms the output matrix C. The feed-through term $\mathbf{D}$ is estimated solving a least squares optimization ${ }^{(22)}$. For the 
VCM modeling, first an estimated second order model $G_{2 n d}$ representing the main actuator dynamics including the low frequency friction mode ${ }^{(24)}$ at $17 \mathrm{~Hz}$ is removed from the FRF measurement and added back to the model after the estimation. The second order model (here given in continuous time) is parameterized by

$$
G_{2 n d}=\frac{K_{v} \omega_{0}^{2}}{s^{2}+2 \delta \omega_{0} s+\omega_{0}^{2}}
$$

and the parameters are given by $K_{v}=9750, \omega_{0}=17 \cdot 2 \pi \frac{\mathrm{rad}}{\mathrm{s}}, \delta=0.2$. The measurements and the estimated models of both actuators are depicted in Fig. 4 and Fig. 5, respectively.
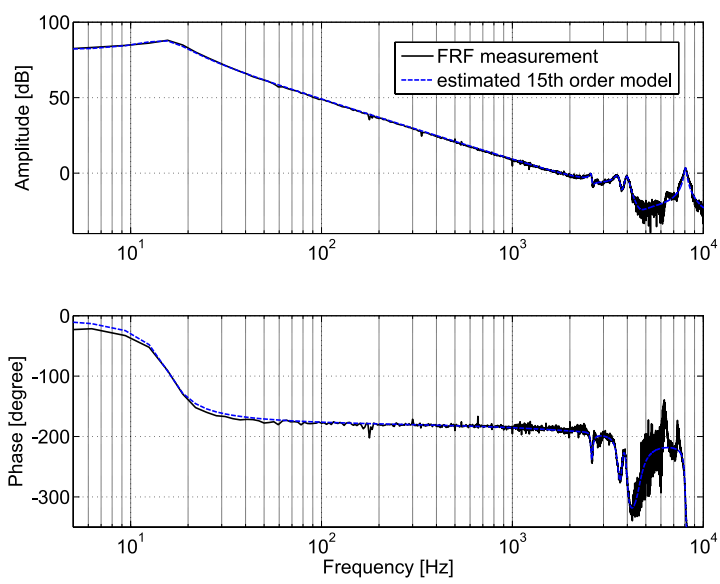

Fig. 4 Comparison of FRF measurement and estimated 15th order VCM model
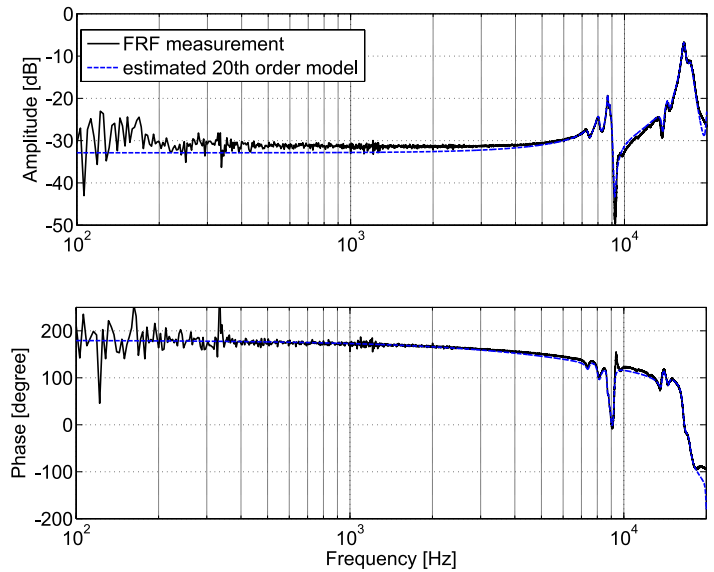

Fig. 5 Comparison of FRF measurement and estimated 20th order PZT model

Furthermore, a unit time-delay was incorporated in the micro-actuator model.

\section{Controller design}

\subsection{General overview}

One of the main characteristics of a dual-stage controller in HDDs is that there are two control outputs but there is only one position feed-back signal available that includes the contribution of both actuators. The relative displacement between the two actuators is not measured in an actual disk drive. Several different control design techniques for dual-stage actuators have been developed in recent years ${ }^{(4)}$. Some of those methods address the problem of actuator saturation $^{(25),(26)}$ and/or include feed-forward control in addition to feed-back control in order to accomplish combined track-following and track-seeking controllers ${ }^{(27)}$. In this study, we focus on feed-back controllers for track-following and short-distance seeks that do not exceed the stoke of the micro-actuator. 
One dual-stage controller design technique is the PQ method ${ }^{(28)}$ that is based on loop shaping. It is shown in ${ }^{(28)}$ that by placing the closed-loop zeros of the feed-back connection of plant $P$ and compensator $Q$ one can achieve frequency separation between both actuators. Here, $P$ is defined as the ratio of the VCM and the PZT model and the compensator $Q$ is defined in the same manner. Application examples of the PQ method are given in $^{(23)}$ and $^{(29)}$. In this study, $P$ yields a non-minimum phase system which limits bandwidth and makes it much more difficult to perform loop shaping based control design. Instead, we applied the sensitivity decoupling method (SDM) as a classical control design technique and an $H_{\infty}$ based optimal control algorithm. Both design methods will be explained briefly in the next two subsections.

\subsection{Sensitivity decoupling method}

The sensitivity decoupling method $(\mathrm{SDM})^{(1),(4),(30)}$ allows a separate controller design for the VCM and the PZT. The control structure is given in Fig. 6. The displacement of the

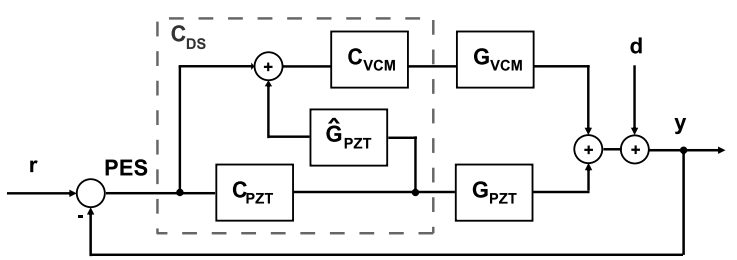

Fig. 6 Control structure of sensitivity decoupling method

PZT is estimated using a simplified PZT model $\hat{G}_{P Z T}$. From Fig. 6, we extract the sensitivity function $S_{T}=\frac{y}{d}$ of the overall system

$$
S_{T}=\underbrace{\frac{1}{1+C_{P Z T} G_{P Z T}}}_{S_{P Z T}} \cdot \underbrace{\frac{1}{1+K \cdot C_{V C M} G_{V C M}}}_{S_{V C M}}
$$

where $G_{(V C M, P Z T)}$ and $C_{(V C M, P Z T)}$ represent the plant dynamics and the controller for both actuators, respectively. A coupling factor $K$ is defined by

$$
K=\frac{1+C_{P Z T} \hat{G}_{P Z T}}{1+C_{P Z T} G_{P Z T}}
$$

where $\hat{G}_{P Z T}$ is a model of the PZT actuator. An obvious choice for $\hat{G}_{P Z T}$ would be the 20th order model depicted in Fig.5. However, to limit the complexity of the controller, $\hat{G}_{P Z T}$ is approximated by a simple DC gain $g_{P Z T}$. The higher frequency resonance modes of the PZT do not have a significant impact on $S_{V C M}$ because of a high frequency roll-off that is included in $C_{V C M}$. Hence, $K \approx 1$ and both control loops can be decoupled and designed separately.

$C_{P Z T}$ is designed as a band pass filter including a notch filter to suppress the microactuator (sway) mode ${ }^{(31)}$ at $17 \mathrm{kHz}$. Thereafter $C_{V C M}$ is designed containing a low pass filter approximating an integrator, a second order lead lag compensator and a high frequency rolloff. The actual dual-stage controller $C_{D S}$ in a classical control loop definition yields

$$
C_{D S}=\left[\begin{array}{c}
\left(1+g_{p Z t} C_{P Z T}\right) C_{V C M} \\
C_{P Z T}
\end{array}\right]
$$

The dual-stage controller is depicted as the solid lines in Fig. 8 where the left plot shows the actual VCM controller $\left(1+g_{p z t} C_{P Z T}\right) C_{V C M}$ and the right plot shows the micro-actuator controller $C_{P Z T}$. The total order of the dual-stage controller is 16 .

\section{3. $H_{\infty}$ loop shaping controller design}

In addition to the sensitivity decoupling controller that is designed using loop shaping techniques only, a combined approach is applied that uses loop shaping and $H_{\infty}$ optimal control design via $H_{\infty}$ loop shaping ${ }^{(32),(33)}$. Figure 7 shows the main principle. Information on 


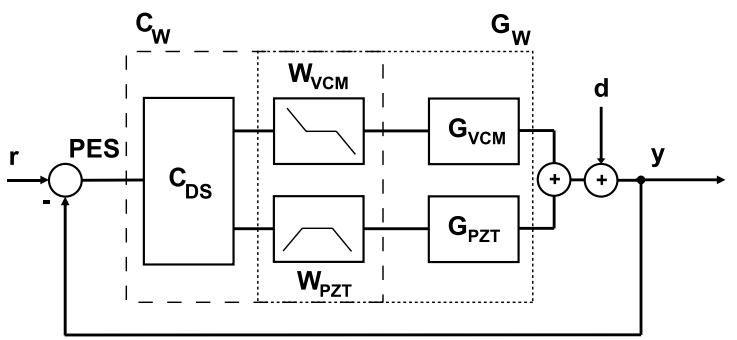

Fig. $7 \quad H_{\infty}$ loop shaping control structure

the $H_{\infty}$ loop shaping algorithm is given in ${ }^{(34)}$. The principle steps of the $H_{\infty}$ loop shaping algorithm are:

First, weighting filters $W_{V C M}$ and $W_{P Z T}$ are designed for both actuator models that represent the shape of the optimal controllers to be estimated. Then, a 4-block $H_{\infty}$ control problem is formulated and used to minimize control signal peaking and error rejection peaking. Given the optimization constraints, an optimal controller $C_{D S}$ is computed. Finally, the weighting filters are preserved in $C_{D S}$.

We define the weighted plant $G_{W}$ (dotted box in Fig. 7) as

$$
G_{W}=\left[\begin{array}{cc}
W_{V C M} & 0 \\
0 & W_{P Z T}
\end{array}\right]\left[\begin{array}{c}
G_{V C M} \\
G_{P Z T}
\end{array}\right]
$$

The weighting functions are defined by

$$
\begin{aligned}
& W_{V C M}=\frac{1}{K_{V C M}} \frac{\tau_{1} s+1}{\tau_{2} s+1} \frac{1}{\tau_{3} s+1} \\
& W_{P Z T}=\frac{R_{g}}{K_{P Z T}} \frac{s}{\tau_{4} s+1} \frac{1}{\tau_{5} s+1}
\end{aligned}
$$

where the design parameters are given by $\frac{1}{\tau_{1}}=2 \pi \cdot 200, \frac{1}{\tau_{2}}=2 \pi \cdot 1, \frac{1}{\tau_{3}}=2 \pi \cdot 5000, \frac{1}{\tau_{4}}=2 \pi \cdot 10$, $\frac{1}{\tau_{5}}=2 \pi \cdot 800 \frac{\mathrm{rad}}{\mathrm{s}}$ and $R_{g}=5$. The gains $K_{V C M}$ and $K_{P Z T}$ are adjusted in such a way that the $0-\mathrm{dB}$ crossover frequency of the weighted plants are located at $500 \mathrm{~Hz}$, respectively, and $R_{g}$ is defined as the relative gain of the PZT with respect to the VCM at the crossover frequency. The $H_{\infty}$-norm of the closed-loop transfer function $T\left(G_{W}, C_{D S}\right)$, defined by

$$
T=\left[\begin{array}{c}
G_{W} \\
I
\end{array}\right]\left[I+C_{D S} G_{W}\right]^{-1}\left[\begin{array}{cc}
C_{D S} & I
\end{array}\right],
$$

is analytically minimized using normalized coprime factorization and a Nehari extension ${ }^{(34)}$. Since the calculated controller is of high order (on the order of the plant), a closed-loop reduction routine that subdivides the high order controller into its low order components is applied. A 10th order stable approximation was obtained and is shown as the dashed lines in Fig. 8.
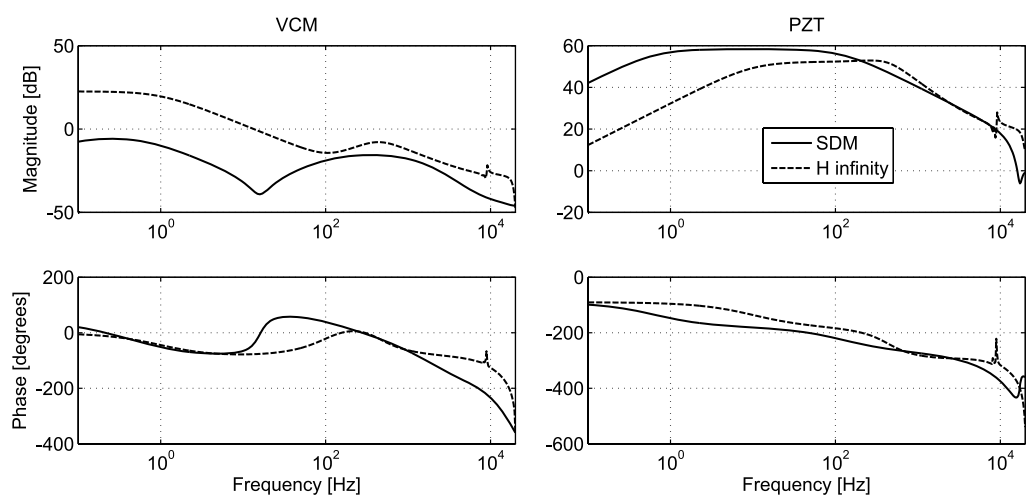

Fig. 8 Comparison SDM and $H_{\infty}$ loop shaping controller 


\subsection{Controller Evaluation}

To evaluate the performance of the designed controllers, the closed loop feed-back connection is simulated. The sensitivity functions for both controllers are shown in Fig. 9. The
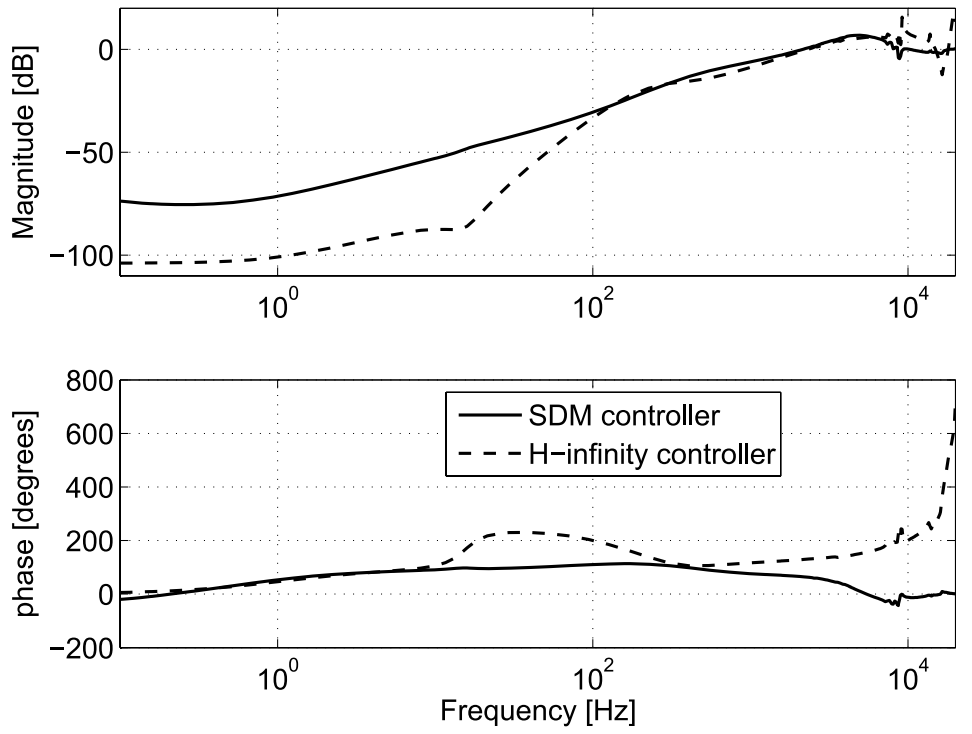

Fig. 9 Comparison of closed loop error rejection (sensitivity function) - $H_{\infty}$ loop shaping controller and sensitivity decoupling method controller

cross-over frequency is nearly the same. However, the $H_{\infty}$ controller shows a better disturbance rejection for lower frequencies than the SDM controller. Another common performance evaluation is a step function as an input representing either a high frequency disturbance or a short track seek. A step size of $100 \mathrm{~nm}$ relates to a track pitch of $250 \mathrm{ktpi}$ in a hard disk drive. Figure 10 shows the simulated response to a step input for both controllers, the control signal for the VCM and the PZT. Furthermore, the individual distribution of the VCM and the PZT to the total displacement is simulated and shown in Fig. 11.
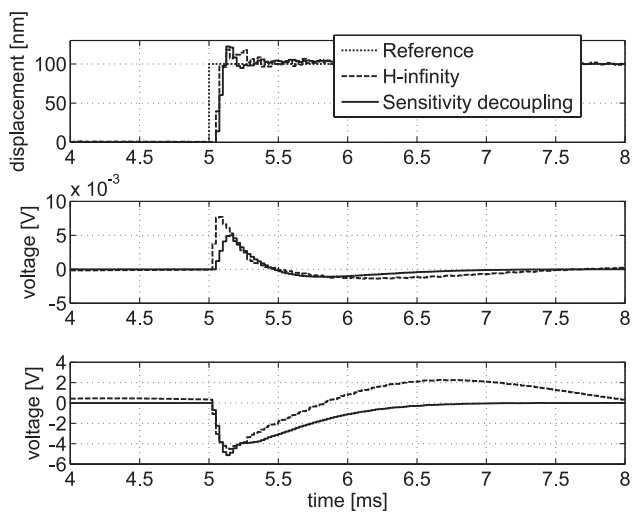

Fig. 10 Simulation of step response - $H_{\infty}$ loop shaping controller and sensitivity decoupling method controller

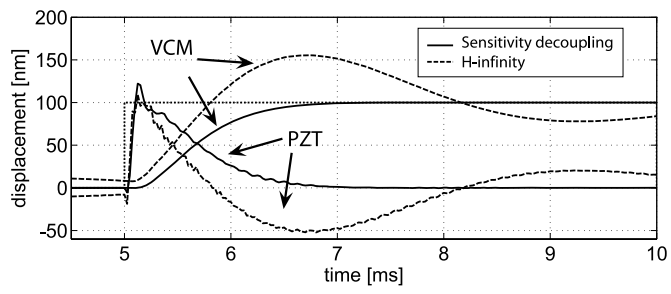

Fig. 11 Simulated displacement for VCM and PZT 
We observe that the SDM controller settles slightly faster than the $H_{\infty}$ controller. Also, the maximum value of the control signal and the overshoot are smaller for the SDM controller. Further performance measures are given in Table 1.

Table 1 Comparison sensitivity decoupling method (SDM) and $H_{\infty}$ loop shaping control design

\begin{tabular}{l|c|c} 
& SDM & $H_{\infty}$ loop shaping \\
\hline gain margin & $6 \mathrm{~dB}$ & $6 \mathrm{~dB}$ \\
phase margin & 54 degrees & 35 degrees \\
overshoot & $22 \%$ & $20 \%$ \\
$10 \%$ settling time & $0.175 \mathrm{~ms}$ & $0.275 \mathrm{~ms}$ \\
crossover frequency & $\approx 2.37 \mathrm{kHz}$ & $\approx 2.32 \mathrm{kHz}$ \\
control signal level & & \\
$\left\|u_{\mathrm{VCM}}\right\|_{\infty}$ & $5 \mathrm{mV}$ & $10 \mathrm{mV}$ \\
$\left\|u_{\mathrm{PZT}}\right\|_{\infty}$ & $5.1 \mathrm{~V}$ & $4.7 \mathrm{~V}$
\end{tabular}
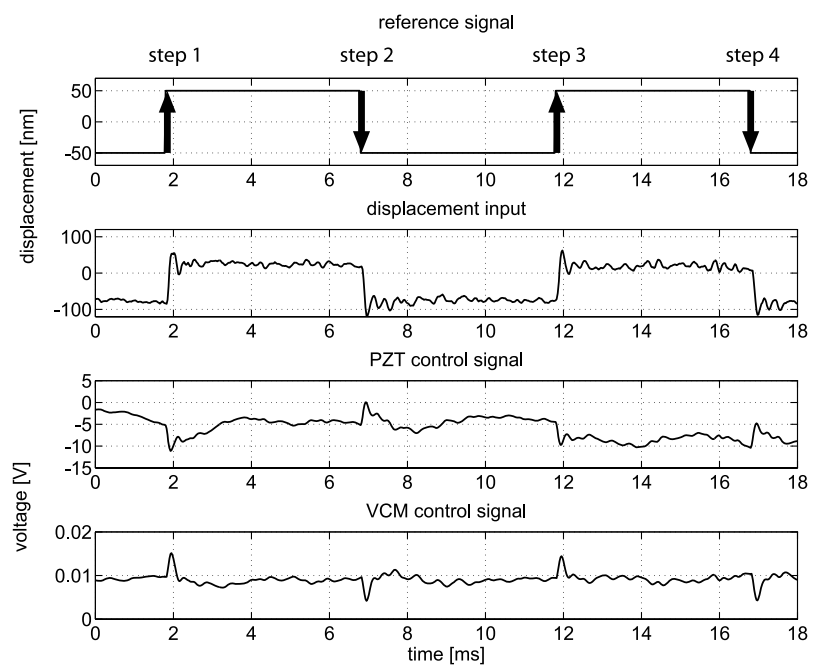

Fig. 12 Implemented sensitivity decoupling method controller for a square wave reference input

\section{Controller implementation}

The controller was implemented at a sampling frequency of $40 \mathrm{kHz}$. A $100 \mathrm{~Hz}$ square wave reference signal was applied representing a number of step functions. The measurement for the SDM controller is shown in Fig. 12. Each rise and fall in the reference signal (indicated by black arrows) is considered as a step and a trigger. Hence, time-based averaging can be applied (see Fig. 13). Numerous oscillations are observed in the averaged measurement. The unaveraged measurement of the $H_{\infty}$ controller implementation is shown in Fig. 14. Looking at the averaged step response (Fig.15), one can observe the same oscillations as in the SDM controller measurement.

The major oscillations in both controller implementations occur at about $2 \mathrm{kHz}$ and $3.5 \mathrm{kHz}$. Furthermore, the control signals show the frequencies of the HDD spindle speed $(167 \mathrm{~Hz})$ and eigenfrequencies. It is conjectured that numerous repeatable (non-stochastic) disturbances that are not affected by time-based averaging cause the vibrations.

\section{Conclusion}

A hard disk drive with dual-stage-suspensions was modified to allow open loop FRF measurements of both servo actuators without having access to the PES. A discrete-time modeling algorithm based on frequency response function measurements was proposed. Two different dual-stage track-following controllers were designed using classic loop shaping techniques combined with modern $H_{\infty}$ control problem algorithms. Both controllers show similar servo 

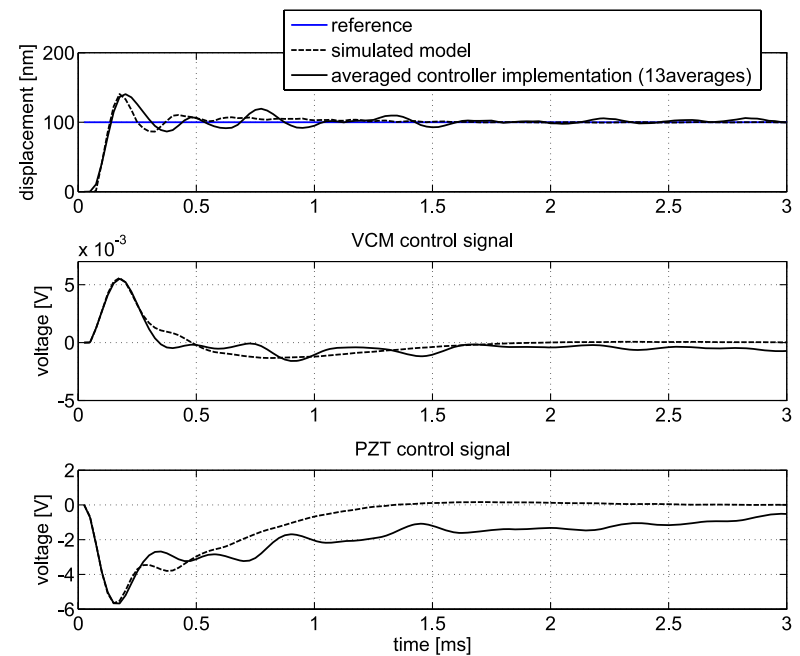

Fig. 13 Head position and control signals for implemented SDM controller (averaged)

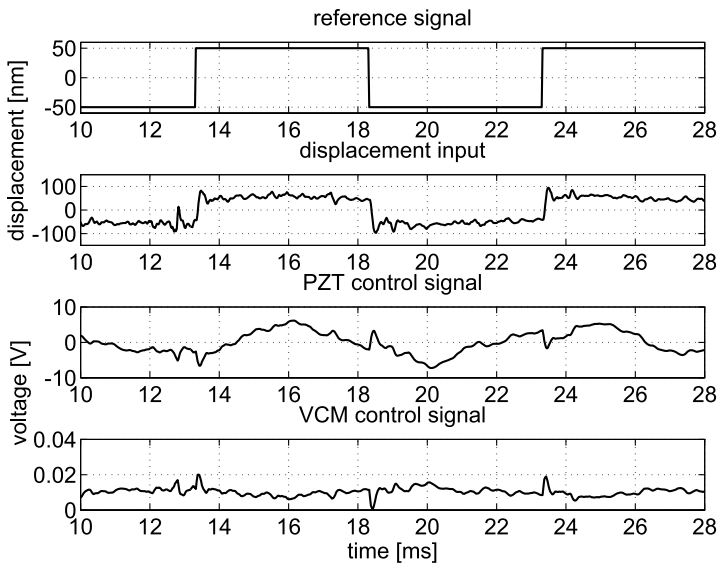

Fig. 14 Implemented $H_{\infty}$ loop shaping controller for a square wave reference input
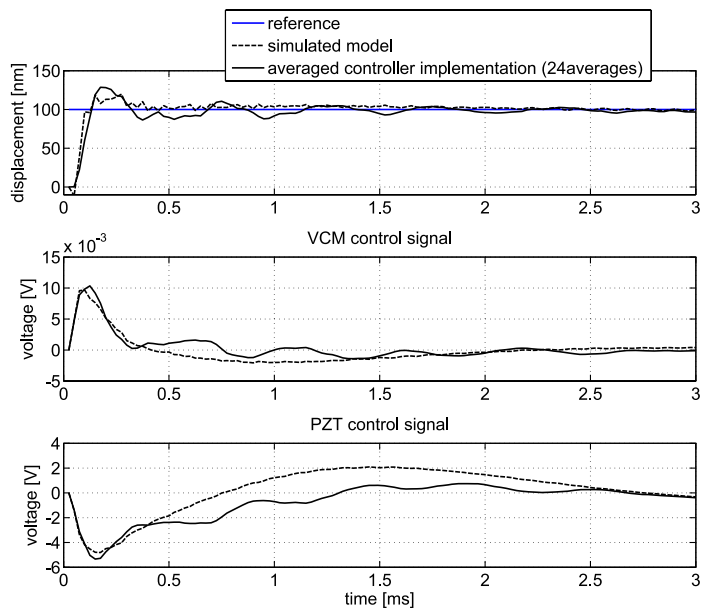

Fig. 15 Head position and control signals for implemented $H_{\infty}$ loop shaping controller (averaged) 
performance. However, the $H_{\infty}$ controller shows a better disturbance rejection than the SDM controller for low frequencies which is due to a high gain in the VCM controller for low frequencies (see Fig.8). Also, the $H_{\infty}$ approach does not use notch filters, and, thus, is more robust against variations in the occurrence of resonance modes than the SDM controller. Since the optimization routine is constrained by the pre-defined parameters in the weighting functions different weighting functions might result in a better controller performance of the $H_{\infty}$ controller.

Both, model estimation and optimized controller design based on predefined controller shape filters can be implemented in the hard disk drive firmware. Since actuator dynamics could be a function of tolerances during manufacturing, the drive could perform a controller calibration itself, and, thus, could improve the servo performance and the TMR budget.

The different controllers designed in this study were implemented in the HDD and showed a stable feed-back control. Small differences between measurement and simulation were observed that are caused by repeatable disturbances.

\section{Acknowledgement}

We would like to thank Seagate Technology, in particular, Alexei Sacks, for his interest in this work and for his help.

\section{References}

( 1 ) K. Mori, T. Munemoto, H. Otsuki, Y. Yamaguch, K. Akagi, "A Dual Stage Magnetic Disk Drive Actuator Using A Piezoelectric Device for a High Track Density", IEEE Trans. on Magnetics, vol. 27(6), pp. 5298-5300, 1991

( 2 ) C.K. Thum,C. Du, J. Zhang, K.P. Tan, B.M. Chen,E.H. Ong, "Servo Control Design for a High TPI Servo Track Writer With Microactuators",IEEE Trans. on Magnetics, vol. 44, no. 9, 2227-2234, 2008

( 3 ) N. Afzulpurkar, Y. Weerakamhaeng, "Precision positioning using MEMS based microactuator", Mechatronics, vol. 12, pp. 1213-1223, 2002

( 4 ) R. Horowitz, Y.Lib, K. Oldhama, S. Kona, X. Huang, "Dual-stage servo systems and vibration compensation in computer hard disk drives", Control Engineering Practice, vol. 15,pp. 291-305, 2007

( 5 ) X. Huang, R. Horowitz, Y. Li, 'Track-following control with active vibration damping and compensation of a dual-stage servo system", Springer Microsyst Technol, vol. 11, pp. 1276-1286, 2005

( 6 ) T. Hirano, L.-S. Fan, W.Y. Lee, J. Hong, W. Imaino, S. Pattanaik, S. Chan, P. Webb, R. Horowitz, S. Aggarwal, D.A. Horsley, "High-Bandwidth High-Accuracy Rotary Microactuators for Magnetic Hard Disk Drive Tracking Servos", IEEE/ASME Trans. on Mechatronics, vol. 3, no. 3, pp. 156-165, 1998

( 7 ) L.-S. Fan, H.H. Ottesen, T.C. Reiley, R.W. Wood, "Magnetic Recording Head Positioning at Very High Track Densities Using a Microactuator-Based, Two-Stage Servo System”, IEEE Trans. on Industial Electronics, vol. 42, no. 3, pp. 222-233, 1995

( 8 ) D.A. Horsley, N. Wongkomet, R. Horowitz, A.P. Pisano, "Precision Positioning Using a Microfabricated Electrostatic Actuator", IEEE Trans. on Magnetics, vol. 35, no. 2, pp. 993-999, 1999

( 9 ) T.Semba, T. Hirano, J.Hongt, L. Fan,’Dual-stage servo controller for HDD using MEMS microactuator", IEEE Trans. on Magnetics, vol. 35, no. 5, pp. 2271-2273, 1999

(10) C.C. Chung, C.W. Seo, S.-H. Lee, "Two Degree-of-Freedom Dual-Stage Actuator Controller Design for Hard Disk Drives”, IEEE Trans. on Magnetics, vol. 36, no. 5, pp. 2255 2257, 2000

(11) Y.Li, F. Marcassa, R. Horowitz, R. Oboe, R. Evans, "Track-Following Control With Active Vibration Damping of a PZT-Actuated Suspension Dual-Stage Servo System”, 
ASME Journal of Dynamic Systems, Measurement and Control, vol. 128, pp. 568-576, 2006

(12) S. Nakamura, H. Numasato, K. Sato, M. Kobayashi, I. Naniwa, "A push-pull multilayered piggyback PZT actuator", Springer Microsystem Technologies, vol.8, pp. 149154, 2002

(13) R.B. Evans, J.S. Griesbach, W.C. Messner, "Piezoelectric Microactuator for Dual Stage Control", IEEE Trans. on Magnetics, vol.35, no. 2, pp. 977-982, 1999

(14) H.H. Gatzen, P.J.P. Freitas,E. Obermeier, J. Robertson, ”A Slider With an Integrated Microactuator (SLIM) for Second Stage Actuation in Hard Disc Drives",IEEE Trans. on Magnetics, vol. 44, no. 11, 3726-3729, 2009

(15) S. Devasia, E. Eleftheriou,S.O. Reza Moheimani, "A Survey of Control Issues in Nanopositioning",IEEE Trans. on Control Systems Technology, vol. 15, no. 5, 802-823, 2007

(16) C.K.Pang, F.L. Lewis, S.S.Ge, G. Guo, B.M. Chen, T.H.Lee, "Singular Perturbation Control for Vibration Rejection in HDDs Using the PZT Active Suspension as Fast Subsystem Observer",IEEE Trans. on Industrial Electronics, vol. 54, no. 3, 1375-1386, 2007

(17) C.K. Pang,G. Guo,B.M. Chen,T.H.Lee, "Self-Sensing Actuation for Nanopositioning and Active-Mode Damping in Dual-Stage HDDs",IEEE Trans. on Mechatronics, vol. 11, no. 3, 328-338, 2006

(18) Chen, B.M., Lee, T.H., Peng, K., Venkataramanan, V., 2006, Hard Disk Drive Servo Systems, 2nd ed., Springer-Verlag, ISBN: 978-1-84628-304-8

(19) B. Hredzak, G. Herrmann, G. Guo, "A Proximate-Time-Optimal-Control Design and Its Application to a Hard Disk Drive Dual-Stage Actuator System",IEEE Trans. on Magnetics, vol. 42, no. 6, 1708-1715, 2006

(20) P.A. Ioannou, H. Xu, B. Fidan, 'Identification and High Bandwidth Control of Hard Disk Drive Servo Systems Based on Sampled Data Measurements",IEEE Trans. on Control Systems Technology, vol. 15, no. 6, 1089-1095, 2007

(21) J.N. Juang, R.S. Pappa, "Eigensystem realization algorithm for modal parameter identification and model reduction", Journal of guidance, control, and dynamics, vol. 8, issue 5, p620, 1985

(22) M. Claes, M.R. Graham, R.A. de Callafon, "Frequency domain subspace identification of a tape servo system", Springer Microsystem Technologies, 2007 13: 1439-1447

(23) U. Boettcher, B. Raeymaekers, R.A. de Callafon, F.E. Talke, "Dynamic modeling and control of a piezo-electric dual-stage tape servo actuator", IEEE Transactions on Magnetics, accepted for publication Jan 2009

(24) A. Mamum,G. Guo,C.Bi, 2007, Hard Disk Drive - Mechatronics and Control, CRC Press, Taylor and Francis Group, Boca Raton, FL, USA

(25) G. Herrmann, B. Hredzak, M.C. Turner, I. Postlethwaite,G. Guo, ”Discrete Robust Anti-Windup to Improve a Novel Dual-Stage Large-Span Track-Seek/Following Method",IEEE Trans. on Control Systems Technology, vol. 16, no. 6, 1342-1351, 2008

(26) D. ,Wu, G. Guo, "Reset Integral-Derivative Control for HDD Servo Systems",IEEE Trans. on Control Systems Technology, vol. 15, no. 1, 161-167, 2007

(27) J.Zhang, C.Du, S. Sam Ge, "A Novel Settling Controller for Dual-Stage Servo Systems",IEEE Trans. on Magnetics, vol. 44, no. 11, 3757-3760, 2008

(28) S.J. Schroeck, W.C. Messner, ”On controller design for linear time-invariant dual-input single-output systems", Proceedings of the American Control Conference, San Diego, CA, USA, 1999

(29) M. Graham, R.J.M. Oosterbosch, R.A.de Callafon, "Fixed order PQ-control design method for dual stage instrumented suspension”, IFAC Congress, Praha, Czech Republic, 2005

(30) M. Kobayashi, R. Horowitz, "Track Seek Control for Hard Disk Dual-Stage Servo Sys- 
tems",IEEE Trans. on Magnetics, vol. 37, no. 2, 949-954, 2001

(31) N.F. Gunderson, K.J. Schulz, T.A. Bordson et al., "Component Development and Product Integration of Secondary Piezoelectric Actuator for Disc Drives", ASME/JSME Joint Conference on Micromechatronics for Information and Precision Equipment, Santa Clara, CA, U.S.A., June 21-23, 2006

(32) D. McFarlane, K. Glover, "A Loop Shaping Design Procedure Using $H_{\infty}$ Synthesis", IEEE Transactions on Automatic Control, vol. 37, no. 6, 759-769, 1992

(33) K. Glover, D. McFarlane, "Robust Stabilization of Normalized Coprime Factor Plant Descriptions with $H_{\infty}$-Bounded Uncertainty", IEEE Transactions on Automatic Control, vol. 34, no. 8,821-830,1989

(34) P.M.M. Bongers, O.H. Bosgra, "Low Order Robust $H_{\infty}$ Controller Synthesis", Proceedings of the 29th Conference on Decision and Control, Honululu, Hawaii, 1990 